\title{
Tumor cell-adipocyte gap junctions activate lipolysis and are essential for breast tumorigenesis
}

\author{
Roman Camarda ${ }^{1,2}$, Jeremy Williams ${ }^{1,2}$, Serghei Malkov ${ }^{3}$, Lisa J. Zimmerman ${ }^{4,5}$, Suzanne Manning ${ }^{6}$, Dvir \\ $\operatorname{Aran}^{7}$, Andrew Beardsley ${ }^{1,8}$, Daniel Van de Mark ${ }^{1}$, Yong Chen ${ }^{1,9,10}$, Charles A. Berdan ${ }^{11,12,13}$, Sharon M. \\ Louie $^{11,12,13,19}$, Celine Mahieu ${ }^{1}$ Juliane Winkler ${ }^{14,15}$, Elizabeth Willey 14,15,20, John D. Gagnon ${ }^{2,16,17}$, Kosaku \\ Shinoda ${ }^{1,9,10}$, K. Mark Ansel ${ }^{16,17}$, Zena Werb ${ }^{14,15}$, Daniel K. Nomura ${ }^{11,12,13}$, Shingo Kajimura ${ }^{1,9,10}$, Atul J. \\ Butte $^{7}$, Melinda E. Sanders ${ }^{6}$, Daniel C. Liebler ${ }^{4,5}$, Hope Rugo ${ }^{8}$, Gregor Krings ${ }^{18}$, John A. Shepherd ${ }^{3,21}$, and \\ Andrei Goga ${ }^{1,8,15 *}$
}

${ }^{1}$ Department of Cell \& Tissue Biology, University of California, San Francisco, San Francisco, CA, USA.

${ }^{2}$ Biomedical Sciences Graduate Program, University of California, San Francisco, San Francisco, CA, USA.

${ }^{3}$ Department of Radiology \& Biomedical Imaging, University of California, San Francisco, San Francisco, CA, USA.

${ }^{4}$ Department of Biochemistry, Vanderbilt University School of Medicine, Nashville, TN, USA.

${ }^{5} \mathrm{Jim}$ Ayers Institute for Precancer Detection and Diagnosis, Vanderbilt-Ingram Cancer Center, Nashville, TN, USA.

${ }^{6}$ Department of Pathology, Vanderbilt University School of Medicine, Nashville, TN, USA.

${ }^{7}$ Institute for Computational Health Sciences, University of California, San Francisco, San Francisco, CA, USA.

${ }^{8}$ Department of Medicine, University of California, San Francisco, San Francisco, CA, USA.

${ }^{9}$ Diabetes Center, University of California, San Francisco, San Francisco, CA, USA.

${ }^{10}$ Eli and Edythe Broad Center of Regeneration Medicine and Stem Cell Research, University of California, San Francisco, San

Francisco, CA, USA.

${ }^{11}$ Department of Chemistry, University of California, Berkeley, Berkeley, CA, USA.

${ }^{12}$ Department of Molecular \& Cell Biology, University of California, Berkeley, Berkeley, CA, USA.

${ }^{13}$ Department of Nutritional Sciences \& Toxicology, University of California, Berkeley, Berkeley, CA, USA.

${ }^{14}$ Department of Anatomy, University of California, San Francisco, San Francisco, CA, USA.

${ }^{15}$ Helen Diller Family Comprehensive Cancer Center, University of California, San Francisco, San Francisco, CA, USA.

${ }^{16}$ Department of Microbiology \& Immunology, University of California, San Francisco, San Francisco, CA, USA.

${ }^{17}$ Sandler Asthma Basic Research Center, University of California, San Francisco, San Francisco, CA, USA.

${ }^{18}$ Department of Pathology, University of California, San Francisco, San Francisco, CA, USA.

Current address:

${ }^{19}$ Department of Genetics, Harvard Medical School, Boston, MA, USA

${ }^{20}$ Biologie Moleculaire et Cellulaire, Universite Pierre et Marie Curie, Paris, France

${ }^{21}$ Population Sciences in the Pacific Program, University of Hawaii Cancer Center, Honolulu, HI, USA

*Correspondence to: Andrei.Goga@ucsf.edu

\section{Abstract}

During tumorigenesis, a heterotypic interface exists between cancer and stromal cells that can both support and repress tumor growth. In the breast, studies have demonstrated a pro-tumorigenic role for adipocytes. However, the molecular mechanisms by which breast cancer cells coopt adipocytes remain elusive. Studying breast tumors and normal adjacent tissue (NAT) from several patient cohorts and mouse models, we show that lipolysis and lipolytic signaling are activated in NAT. We investigate the tumor-adipocyte interface and find that functional gap junctions form between breast cancer cells and adipocytes. As a result, cAMP, a critical lipolysis-inducing signaling molecule, is transferred from breast cancer cells to adipocytes and activates lipolysis in a gap junction-dependent manner; a fundamentally new mechanism of lipolysis activation in adipocytes. We find that gap junction formation depends upon connexin 31 (Cx31), and that $\mathrm{Cx} 31$ is essential for breast tumor growth and activation of lipolysis in vivo. Thus, direct tumor celladipocyte interaction is critical for tumorigenesis and may serve as a new therapeutic target in breast cancer.

One sentence summary: Gap junctions between breast cancer cells and adipocytes transfer cAMP and activate lipolysis in the breast tumor microenvironment.

Keywords: Breast cancer, triple-negative breast cancer, TNBC, adipocyte, gap junction, lipolysis, cAMP, connexin 31, Cx31, GJB3 
Camarda R, et al. 2018 - preprint version -www.biorxiv.org

\section{Materials and Methods:}

\section{CB imaging protocol}

The 3CB method combines dual-energy X-ray mammography attenuations and a breast thickness map to solve for the three unknowns: water, lipid, and protein content (1). We used Hologic Selenia full-field digital mammography system (Hologic, Inc.) to image women with $3 \mathrm{CB}$. Two dual energy mammograms were acquired on each woman's affected breast using a single compression. The first exposure was made under conditions of regular clinical screening mammogram. The second mammogram was acquired at a fixed voltage $(39 \mathrm{kVp})$ and $\mathrm{mAs}$ for all participants. A high energy exposure $(39 \mathrm{kVp} / \mathrm{Rh}$ filter) was made using an additional 3-mm plate of aluminum in the beam to increase the average energy of the high energy image. We limited the total dose of this procedure to be approximately $110 \%$ of the mean-glandular dose of an average screening mammogram. The images were collected under an investigational review board approval to measure breast composition. The breast thickness map was modeled using the SXA phantom (2). The thickness validation procedure concluded in a weekly scanning of specially designed quality assurance phantom (3). The calibration standards and $3 \mathrm{CB}$ algorithms are described in full elsewhere $(1,4)$. The region of interests of lesions and three surrounding rings of $2 \mathrm{~mm}$ distance outward from lesion boundary were derived for water, lipid, and protein maps. The median lipid measures of regions of interest within lesions, three rings outside of lesions, differences and ratios between lesions and rings were generated for both CC and MLO mammograms. Average values of generated variables of two views were used.

\section{CB patient population}

Five hundred women with suspicious mammography findings (BIRADS 4 or greater) were recruited and imaged before their biopsies using a 3-component decomposition dual-energy mammography protocol (3CB) for a multicenter study with two recruitment sites: The University of California at San Francisco and Moffitt Cancer Center, Tampa, Florida (5). All patients received a biopsy of the suspicious area, and breast biopsies were clinically reviewed by the pathologists. A subset of pathology proven triple-negative $(n=6)$ and receptor-positive $(n=40)$ invasive cancers were selected for this study. All women received both cranio-caudal (CC) and mediolateral-oblique (MLO) views. Exclusion criteria for the study were no prior cancer, biopsies, or breast ipsilateral alterations, and no occult findings. This study was approved by the institutional review board of the respective institutions.

\section{Histological sectioning, hematoxylin and eosin staining, and adipocyte area quantification}

Invasive breast carcinomas were obtained from the Pathology Departments of the University of California San Francisco (San Francisco, CA) and Moffitt Cancer Center (Tampa, FL). The study population included 39 hormone receptor positive tumors (32 ER positive $(+) / \mathrm{PR}+/$ HER2 negative, 2 ER+/PR-/HER2-, 4 $\mathrm{ER}+/ \mathrm{PR}+/ \mathrm{HER} 2+$, and $1 \mathrm{ER}+/ \mathrm{PR}-/ \mathrm{HER} 2+$ ), 6 triple negative (ER-/PR-/HER2-) tumors, and 1 ER-/PR-/HER2+ tumor. Thirtynine tumors were invasive ductal carcinomas and 7 were invasive lobular carcinomas. Tissue was fixed in $10 \%$ formalin and embedded in paraffin, and 4 micron sections were cut for hematoxylin and eosin (H\&E) and immunohistochemical ER, PR, and HER2 staining, as well as HER2 fluorescence in situ hybridization (FISH) for a subset of tumors. ER, PR, and HER2 were scored according to ASCO/CAP guidelines $(6,7)$. An H\&Estained slide demonstrating tumor and sufficient (at least $0.5 \mathrm{~cm}$ ) NAT was chosen from each of 11 tumors with available slides and subjected to whole slide scanning at $400 \times$ magnification using an Aperio XT scanner (Leica Biopsystems, Buffalo Grove, IL). Images were visualized using ImageScope software (Leica Biosystems). For each tumor, 4 representative images at 50X magnification (at least 50 adipocytes per image) from R1 and R3 were analyzed using Fiji imaging software with the open source Adiposoft v1.13 plugin (8).

\section{cAMP-dependent lipolysis signature}

The cAMP-dependent lipolysis gene signature was generated using RNA-seq data of cAMP-treated adipocytes (9). Differentially expressed genes were sorted according to their $P$ value and the top 100 upregulated genes were chosen for the signature. This signature was then used to calculated enrichment scores using the single-set gene set enrichment analysis (ssGSEA) method (10). "cAMP 100 signature" enrichment scores were calculated for a dataset containing multiple samples from multiple regions surrounding breast tumors (11). The dataset includes samples from the tumor itself $(\mathrm{n}=9)$, and NAT $1 \mathrm{~cm}(\mathrm{n}=7), 2$ $\mathrm{cm}(\mathrm{n}=5), 3 \mathrm{~cm}(\mathrm{n}=3)$ and $4 \mathrm{~cm}(\mathrm{n}=4)$ away from the tumor, in addition to healthy normal samples $(\mathrm{n}=10)$. The spatial data set of multiple regions surrounding breast tumors was download from EMBL-EBI ArrayExpress (Accession E-TABM-276). Raw CEL files were downloaded and processed using custom Affymetrix GeneChip Human Genome U133 Plus 2.0 CDF obtained from BrainArray (12). The processing and normalization were performed using the Robust Multi-array Average (RMA) procedure on Affymetrix microarray data.

\section{Laser Capture Microdissection}

Breast tumor tissue was sectioned at $6 \mu \mathrm{m}$ in a Leica CM 1850 Cryostat (Leica Microsystems $\mathrm{GmbH}$ ). The sections were mounted on uncharged glass slides without the use of embedding media and placed immediately in $70 \%$ ethanol for 30 seconds. Subsequent dehydration was achieved using graded alcohols and xylene treatments as follows: $95 \%$ ethanol for 1 minute, $100 \%$ ethanol for 1 minute (times 2), xylene for 2 minutes and second xylene 3 minutes. Slides were then dried in a laminar flow hood for 5 minutes prior to microdissection. Then, sections were laser captured microdissected with PixCell II LCM system (Arcturus Engineering). Approximately 5000 shots using the 30 micron infrared laser beam will be utilized to obtain approximately 10,000 cells per dissection. All samples were microdissected in duplicate on sequential sections.

\section{SDS-PAGE and In-gel Digestion}

All membranes containing the microdissected cells from breast tumor tissue were removed and placed directly into a $1.5 \mathrm{~mL}$ Eppendorf tube. Membranes containing the microdissected cells were suspended in $20 \mu \mathrm{L}$ of SDS sample buffer, reduced with 
DTT and heated in a 70-80C water bath for approximately $10 \mathrm{~min}$. The supernatant was then electrophoresed approximately $2 \mathrm{~cm}$ into a $10 \%$ Bis Tris gel, stained with Colloidal Blue with destaining with water, and the region was excised and subjected to in-gel trypsin digestion using a standard protocol. Briefly, the gel regions were excised and washed with $100 \mathrm{mM}$ ammonium bicarbonate for 15 minutes. The liquid was discarded and replaced with fresh $100 \mathrm{mM}$ ammonium bicarbonate and the proteins reduced with $5 \mathrm{mM}$ DTT for 20 minutes at $55^{\circ} \mathrm{C}$. After cooling to room temperature, iodoacetamide was added to $10 \mathrm{mM}$ final concentration and placed in the dark for 20 minutes at room temperature. The solution was discarded and the gel pieces washed with $50 \%$ acetonitrile $/ 50 \mathrm{mM}$ ammonium bicarbonate for 20 minutes, followed by dehydration with $100 \%$ acetonitrile. The liquid was removed and the gel pieces were completely dried, reswelled with $0.5 \mu \mathrm{g}$ of modified trypsin (Promega) in $100 \mathrm{mM}$ $\mathrm{NH}_{4} \mathrm{HCO}_{3}$, and digested overnight at $37^{\circ} \mathrm{C}$. Peptides were extracted by three changes of $60 \%$ acetonitrile $/ 0.1 \%$ TFA, and all extracts were combined and dried in vacuo. Samples were reconstituted in $35 \mu \mathrm{L} 0.1 \%$ formic acid for LC-MS/MS analysis.

LC-MS/MS Analysis, Protein Identification and Quantitation Peptide digests were analyzed on a Thermo LTQ Orbitrap Velos ion trap mass spectrometer equipped with an Eksigent NanoLC 2D pump and AS-1 autosampler as described previously (13). Peptide sequence identification from MS/MS spectra employed the RefSeq Human protein sequence database, release version 54, and both database and peptide library search strategies (13). For initial protein assembly, peptide identification stringency was set at a maximum of $1 \%$ reversed peptide matches, i.e., $2 \%$ peptideto-spectrum matches (PSM) FDR and a minimum of 2 unique peptides to identify a given protein within the full data set. To minimize false-positive protein identifications, only proteins with a minimum of 6 matched spectra were considered. The full dataset contained 850,847 filtered spectra corresponding to 31,594 distinct spectrum-peptide sequence matches, which mapped to 24,946 distinct peptide sequences and 2,230 indistinguishable protein identifications. The protein-level FDR for the final assembly was $5.14 \%$. Spectral counts for each protein in the final assembly were calculated as the sum of peptide-spectrum matches that met the criteria described above.

\section{Orthotopic xenograft studies}

The human samples used to generate patient-derived xenograft (PDX) tumors, as well as the human non-tumor samples, were previously described (14). The generation of the MTBTOM tumor model has been previously described (15). 4-week-old WT FVB/N and immunocompromised NOD/SCID-gamma (NSG) female mice were purchased from Taconic Biosciences. Viably frozen MTBTOM, HCI002, HCI009 and HCI010 tumor samples were transplanted into the cleared mammary fat pads of FVB/N and NSG mice, respectively. Tumor growth was monitored daily by caliper measurement in two dimensions. When tumors reached $1 \mathrm{~cm}$ (MTBTOM) or $2 \mathrm{~cm}$ (PDX) in any dimension mice were euthanized, tumor and NAT were isolated, and flash-frozen in liquid nitrogen. The protocols described in this and other sections regarding animal studies were approved by the UCSF Institutional Animal Care and Use Committee. For the HCC1143 and HS578T control and Cx31 partial knockout orthotopic xenografts, $5 \times 10^{5}$ cells were resuspended 1:1 with matrigel (Corning) and injected into the cleared mammary fat pads of 4-week-old WT NSG female mice. Tumor incidence and growth were monitored daily via palpation and caliper measurement, respectively. Mice were euthanized after 180 days or after tumors reached $2 \mathrm{~cm}$ in any dimension. For HCC1143 $\mathrm{GJB}^{+/+/+}$and $\mathrm{GJB}^{+/+/-}$xenografts, a central slice of tumor and surrounding NAT from separate was fixed in $4 \%$ paraformaldehyde and embedded in paraffin for histological sectioning, H\&E staining and adipocyte area quantification, while the remaining tumor and NAT tissues were flash-frozen in liquid nitrogen. For other xenografts, NAT was isolated and flash-frozen in liquid nitrogen. For the CL316273 experiment, mice were randomized into experimental groups immediately post-orthotopic transplant. The following day, drug treatment was initiated and mice received vehicle or $1 \mathrm{mg} / \mathrm{kg}$ CL316273, delivered by intraperitoneal injection, daily until tumor incidence was recorded via palpation.

\section{Immunoblot analysis}

Proteins were extracted using RIPA buffer (Thermo) and proteinase (Roche) plus phosphatase (Roche) inhibitor cocktails. Protein extracts were resolved using 4-12\% SDS-PAGE gels (Life Technologies) and transferred to nitrocellulose membranes (Life Technologies). Membranes were probed with primary antibodies overnight on a $4{ }^{\circ} \mathrm{C}$ shaker, then incubated with horseradish peroxidase (HRP)-conjugated secondary antibodies, and signals were visualized with ECL (Bio-Rad). The primary antibodies targeting the following proteins were used: $\beta$-actin (actin) (sc-47778 HRP, Santa Cruz, 1:10,000), pHSL S563 (4139, Cell Signaling, 1:1000), HSL (4107, Cell Signaling, 1:1000), HNF4 $\alpha$ (ab41898, Abcam, 1:1000), and Cx31 (ab156582, Abcam, 1:1000). Chemiluminescent signals were acquired with the Bio-Rad ChemiDoc XRS+ System equipped with a supersensitive CCD camera. Where indicated, unsaturated band intensities were quantified using Bio-Rad Image Lab software.

\section{Cell culture and virus production}

A panel of established TN and RP human breast cancer cell lines, and their culture conditions, have previously been described (16). No cell line used in this paper is listed in the database of commonly misidentified cell lines that is maintained by the International Cell Line Authentication Committee (ICLAC) (http://iclac.org/databases/cross-contaminations/). All lines were found to be negative for mycoplasma contamination. Lentiviruses for Cas9 and sgRNAs were produced in 293T cells using standard polyethylenimine (Polysciences Inc.) transfection protocols.

\section{Dye transfer and FACS analysis}

For cancer cell-cancer cell transfer, monolayers of indicated lines (donors) were labelled with $1 \mu \mathrm{M}$ CalceinAM dye (Life Technologies) at $37^{\circ} \mathrm{C}$ for $40 \mathrm{~min}$. Dye-loaded cells were washed three times with PBS, and then single-cell suspensions of $1.5 \mathrm{X}$ $10^{5}$ mCherry-labelled cells (recipients) were added for 5 hours. Dye transfer was quantified by BD LSRFORTESSA or BD LSR II (BD Biosciences). For cancer cell-adipocyte transfer, monolayers of indicated control or Cx31 partial knockout lines (donors) were labelled with $1 \mu \mathrm{M}$ CalceinAM dye at $37^{\circ} \mathrm{C}$ for 40 
Camarda R, et al. 2018 - preprint version -www.biorxiv.org

min. Dye-loaded cells were washed three times with PBS, and then primary mammary adipose tissues (recipient) were added for 5 hours. Primary adipose tissue was isolated from co-culture, washed with PBS, and dye transfer was quantified by measurement of total adipose fluorescence using a Tecan fluorescent plate reader.

\section{Gene expression analysis}

TCGA breast-invasive carcinoma data set was sourced from data generated by TCGA Research Network (cancergenome.nih.gov), made available on the University of California, Santa Cruz (UCSC) Cancer Browser. For the MTBTOM data set 11 endpoint MTBTOM orthotopic xenografts generated as described above and 3 mammary glands from naïve mice were flash-frozen in liquid nitrogen. RNA was isolated using the RNAeasy kit (Qiagen). Library preparation and Illumina RNAseq was performed by $\mathrm{Q}^{2}$ Solutions (www.q2labsolutions.com). All gene expression analyses were performed using the 'limma' R package (17).

\section{ATP quantification}

To determine the effects of CBX treatment on ATP levels, tumor cells were seeded in 96-well plates at 5,000-7,000 cells per well and cultured in the presence of 0 or $150 \mu \mathrm{M} \mathrm{CBX}$ (Sigma) for 48 hours, with triplicate samples for each condition. Relative ATP concentrations were determined using the CellTiter-Glo Luminescent Cell Viability Assay (Promega).

\section{Isolation of primary mammary adipose tissue}

Anonymous reduction mammoplasty samples were acquired from the Cooperative Human Tissue Network (CHTN). Samples were washed in DPBS supplemented with $1 \%$ Penicillin/Streptomycin and $0.1 \%$ Gentamicin (all GIBCO). Mammary adipose tissue was separated mechanically from epithelial tissue using a razor blade and was then cryopreserved in freezing medium (10\% DMSO (Sigma) in FBS (X\&Y Cell Culture)).

\section{Immunofluorescence staining and microscopy}

For adipose tissue-cancer cell co-culture, 1 X $10^{6}$ of the indicated mCherry-labelled cell line was injected into primary mammary adipose tissue and cultured at $37^{\circ} \mathrm{C}$ for 18 hours. The co-cultures were examined using fluorescent microscopy to identify regions of adipose tissue containing mCherry-positive cancer cells. These regions were isolated and fixed in 4\% paraformaldehyde and embedded in paraffin. Primary TNBCs used for immunofluorescence were identified and retrieved from the clinical archives of the University of California San Francisco (UCSF) Department of Pathology. All tumors consisted of estrogen receptor (ER)-, progesterone receptor (PR)-, and HER2negative invasive ductal carcinomas. Breast tissue was fixed in $10 \%$ formalin and embedded in paraffin. Tumor blocks with sufficient tumor and adjacent (at least $0.5 \mathrm{~cm}$ ) normal tissue were selected, and $4 \mu \mathrm{m}$ sections were cut on plus-charged slides for immunofluorescence. This study was approved by the UCSF institutional review board. For immunofluorescence labeling, slides were dewaxed in xylene followed by rehydration in graded ethanol $(100,95,70 \%)$ and deionized H2O. Antigen retrieval was performed in 10mM Tris, $1 \mathrm{mM}$ EDTA, $0.05 \%$ Tween 20, $\mathrm{pH} 9$ at
$121{ }^{\circ} \mathrm{C}$ for $4 \mathrm{~min}$. Subsequently, tissue sections were blocked in $1 \%$ bovine serum albumin, $2 \%$ fetal bovine serum in PBS for 5 min, and incubated with primary antibodies (Cx31, 12880, Proteintech, 1:50 and pan-cytokeratin, sc-81714, Santa Cruz, 1:50) overnight at $4{ }^{\circ} \mathrm{C}$. Following several PBS washes, sections were incubated with Alexa Fluor-488 or -568 conjugated antibodies, counterstained with DAPI (Sigma), and mounted using Vectashield (Vector). Epifluorescence images were acquired either by spinning disk microscopy on a customized microscope setup as previously described (18-20) except that the system was upgraded with a next generation scientific CCD camera (cMyo, 293 Photometrics) with $4.5 \mu \mathrm{m}$ pixels allowing optimal spatial sampling using a $\AA \sim 60$ NA 1.49 objective (CFI 294 APO TIRF; Nikon), or at the UCSF Nikon Imaging Center using a Nikon Ti Microscope equipped with an Andor Zyla 5.5 megapixel sCMOS camera and Lumencor Spectra-X 6-channel LED illuminator. Images were collected using a Plan Apo $\lambda 20 \mathrm{x} /$ 0.75 lens.

\section{Generation of Cx31 partial knockout lines}

LentiCas9-Blast (Addgene plasmid \#52962) and lentiGuide-Puro (Addgene plasmid \#52963) were gifts from Feng Zhang. sgRNAs against Cx31 were constructed using the Feng Zhang Lab CRISPR Design Tool (crispr.mit.edu). sgRNAs used were as follows:

Cx31 exon $1 \mathrm{sg} 1$ : CCAGATGCGCCCGAACGCTGTGG

(HS578T 1-GJB3 $^{+/+/-}$and HCC1143 $\mathrm{GJB}^{+/+/}$)

Cx31 exon $1 \mathrm{sg} 2$ : CCGGGTGCTGGTATACGTGGTGG (HS578T 2-GJB3 $^{+/+/}$and HCC1143 $\mathrm{GJB3}^{+/ /-}$)

Lentiviral transduction was performed in DMEM supplemented with $10 \%$ FBS and polybrene $10 \mu \mathrm{g} / \mathrm{mL}$. Cas9-expressing cells were enriched by Blasticidin (10-15 $\mu \mathrm{g} / \mathrm{mL}$ Gemini BioProducts) selection for seven days. Cas9+ cells were subsequently transduced with lentiGuide-Puro (with sgRNAs targeting Cx31) followed by puromycin $(1 \mu \mathrm{g} / \mathrm{mL}$; Gibco) for seven days. Thereafter, clonal selection was performed and clones screened for loss of target gene protein expression by immunoblot analysis.

\section{cAMP quantification}

For in vitro studies, tumor cells were seeded in 96-well plates at $5,000-7,000$ cells per well and cultured in the presence of 0 or 150 $\mu \mathrm{M}$ CBX (Sigma) for 24 hours, with triplicate samples for each condition. Changes in cAMP concentration were determined using the cAMP-Glo Assay (Promega). For in vivo studies, frozen tissue was homogenized using a TissueLyser in $300 \mu 1$ of 40:40:20 acetonitrile:methanol:water with the addition of $1 \mathrm{nM}$ (final concentration) of $\mathrm{D} 3-[15 \mathrm{~N}]$ serine as an internal extraction standard (Cambridge Isotopes Laboratories Inc, DNLM-6863). 10 $\mu 1$ of cleared supernatant (via centrifugation at 15,000 r.p.m., 10 min, at $4{ }^{\circ} \mathrm{C}$ ) was used for SRM-LC-MS/MS using a normalphase Luna NH2 column (Phenomenex). Mobile phases were buffer A (composed of $100 \%$ acetonitrile) and buffer B (composed of 95:5 water:acetonitrile). Solvent modifiers were $0.2 \%$ ammonium hydroxide with $50 \mathrm{mM}$ ammonium acetate for negative ionization mode. cAMP levels were analyzed using the MassHunter software package (Agilent Technologies) by quantifying the transition from parent precursor mass to product ions. 


\section{cAMP transfer}

For cancer cell-adipocyte transfer, monolayers of indicated control or Cx31partial knockout lines (donors) were labelled with $2 \mu \mathrm{M}$ fluo-cAMP (Biolog Life Science Institute) at $37^{\circ} \mathrm{C}$ for 30 min. cAMP-loaded cells were washed three times with PBS, and then primary mammary adipose tissues (recipient) were added for 5 hours. Primary adipose tissue was isolated from co-culture, washed with PBS, and cAMP transfer was quantified by measurement of total adipose fluorescence using a Tecan fluorescent plate reader.

\section{Preadipocyte differentiation and qRT-PCR}

Primary mouse preadipocytes were differentiated as previously described (21). Monolayers of differentiated adipocytes were washed with PBS, and then treated with vehicle or $10 \mu \mathrm{M}$ forskolin (Sigma), or seeded with $1 \times 10^{5}$ of the indicated cancer lines. Total RNA was isolated from co-cultures after 20 hours using the RNeasy kit (Qiagen). One $\mu \mathrm{g}$ of total RNA was reverse transcribed using iScript cDNA synthesis kit (Bio-Rad). The relative expression of UCP1, aP2, and GAPDH was analyzed using a SYBR Green Real-Time PCR kit (Thermo) with an Applied Biosystems QuantStudio 6 Flex Real-Time PCR System thermocycler (Thermo). Variation was determined using the $\Delta \Delta \mathrm{CT}$ method (22) with GAPDH mRNA levels as an internal control. Mouse-specific primers used were as follows:

GAPDH forward CCAGCTACTCGCGGCTTTA reverse GTTCACACCGACCTTCACCA

UCP1 forward CACCTTCCCGCTGGACACT reverse CCCTAGGACACCTTTATACCTAATGG

aP2 forward ACACCGAGATTTCCTTCAAACTG reverse CCATCTAGGGTTATGATGCTCTTCA

\section{Proliferation assays}

To determine the effects of $\mathrm{Cx} 31$ partial knockout on cell proliferation, the indicated cell lines were seeded in 6-well plates at $1.5 \times 10^{5}$ cells/well. Cells were harvested at 24,48 and $72 \mathrm{~h}$. Cell counts were determined using the Countess Automated Cell Counter (Life Technologies) according to the manufacturer's instructions.

\section{Statistical analysis}

Prism software was used to generate and analyze Spearman correlation (Fig. 1D) and the survival plots (Fig. 4B). Correlation $P$ values were generated using a two-sided t-test. Survival plot $P$ values was generated using a log-rank test. All differential expression analyses (Fig. 2, C and D) were done using the 'limma' R package (17).

\section{Code availability.}

Publicly available data sets were acquired as noted. Our annotations of the TCGA data set is available at (https://bitbucket.org/jeevb/brca).

\section{Main text:}

A variety of cancers, including those of the breast, arise near or within adipose tissue depots (23). Therefore, during tumor development in these organs a heterotypic cell-cell interface exists between adipocytes and cancer cells. We and others have demonstrated that triple-negative breast cancers (TNBC, estrogen/progesterone/HER2 receptor-negative) utilize and require fatty acid oxidation to fuel bioenergetic metabolism (24, 25 ). The origin of the fatty acids being oxidized remains largely unclear. Several studies have demonstrated that tumor cells can secrete cytokines such as tumor necrosis factor- $\alpha$ that induce lipolysis in adjacent adipocytes, and that adipocyte-derived fatty acids can be taken up and oxidized by cancer cells (26-31). These studies, however, have mostly relied upon transwell co-culture methods that do not recapitulate the direct cell-cell contact observed in vivo (27-30). Furthermore, evidence of enhanced lipolysis in adipocytes adjacent to breast tumors has not been well established in clinical patient samples. Mammary adipocytes undergo enhanced lipolysis when in close proximity to non-tumor epithelial cells, suggesting that local pro-lipolytic mechanisms exist, but have yet to be identified $(31,32)$. Thus, we set out to study the breast cancer-adipocyte interface and determine the contribution of cell-cell contact to tumorigenesis.

To determine if lipolysis occurs in normal tissue adjacent to breast tumors (NAT), we employed four independent strategies. First, we employed three-component breast (3CB) composition measure, a radiographic imaging method derived from dualenergy mammography that allows water, lipid and protein content of a tissue to be quantified (1). We postulated that if tumors induce lipolysis in adipocytes, we would observe differences in lipid content between NAT near the tumor compared to NAT further away. Using $3 \mathrm{CB}$ imaging, we assessed the lipid content of clinical breast tumors and the first $6 \mathrm{~mm}$ of NAT segmented into $2 \mathrm{~mm}$ "rings" from 46 patients with invasive breast cancer (Fig. 1A and Table S1). As we have previously demonstrated (5), we found a significant decrease in lipid content in lesions compared to NAT 0-2 mm away (R1) (Fig. 1B). This is congruent with breast tumors being epithelial in nature, while the major constituent of normal breast is adipose tissue (32). Remarkably, we also found that within NAT there was a significant stepwise decrease in lipid content comparing R3 (4-6 mm) to R2 (2-4 mm), and R2 to R1 (Fig. 1B). In addition, we asked whether changes in lipid content between R3 and R1 NAT correlate with receptor status or tumor grade (Table S1). We found that NAT surrounding triple-negative (TN) and grade 2/3 tumors trended towards a greater average change in lipid content than receptor-positive (RP) and grade 1 tumors, respectively (Fig. S1, A and B). These data suggest that adipocytes near breast tumors have partially depleted lipid stores, and that TN and higher-grade tumors may induce this phenomenon to a greater degree than RP and lowgrade tumors.

Previous studies have demonstrated that adipocyte size is inversely correlated to lipolysis status in mammary tissue (33). We quantified average adipocyte size in R1 and R3 in 11 of the 46 patients for whom we had access to histological sections of treatment-naïve tumor and NAT at the time of surgical resection (Fig. 1A, Fig. S1C and Table S1). Similar to the change in lipid content observed, we found a significant decrease in adipocyte size in R1 compared to R3 in all patients analyzed, indicating that 
Camarda R, et al. 2018 - preprint version -www.biorxiv.org

adipocytes are smaller when closer to breast tumors (Fig. 1C). Finally, we correlated the change in lipid content and adipocyte size on an individual patient basis. We found a positive correlation $(\mathrm{R}=0.5818, \mathrm{p}=0.0656)$ between the change in lipid content and adipocyte area (Fig. 1D). Taken together, these data suggest adipocytes are smaller and have diminished lipid content when adjacent to breast tumors, two phenotypes that are established indicators of increased lipolysis (33).

Second, we sought to determine if gene expression changes associated with lipolysis were observed in tumor-adjacent adipocytes. To first generate a lipolysis gene expression signature, we identified the 100 most upregulated genes when a differentiated adipocyte cell culture model is stimulated with cAMP, a critical pro-lipolytic signaling molecule (9). We then used a publically available dataset of gene expression data for primary breast tumors as well as matched NAT 1, 2, 3 and $4 \mathrm{~cm}$ away from the tumor. We sought to determine if enrichment of the lipolysis signature occurred in NAT compared to non-tumor breast tissue obtained from healthy individuals using single-set gene set enrichment analysis $(10,11)$. We found a significant elevation of the cAMP-dependent lipolysis signature in tumor and NAT from all regions analyzed compared to control tissue (Fig. $1 \mathrm{E})$. These data indicate that lipolytic signaling is activated in breast-tumor adjacent adipocytes up to $4 \mathrm{~cm}$ away from the primary tumor. Adipose tissue is sparsely innervated and a recent study found that adipocytes can propagate pro-lipolytic sympathetic signals via direct transfer of cAMP through adipocyte-adipocyte gap junctions (34). Thus, tumor-adjacent adipocytes receiving a pro-lipolytic stimulus may disperse this signal to distant adipocytes via gap junctions, which might explain the elevation of cAMP signaling we observed up to $4 \mathrm{~cm}$ away from the tumor (Fig. 1E).

Third, we sought to determine if there are changes in protein abundance in tumor-adjacent NAT indicative of lipolysis activation. We conducted laser capture microdissection (LCM, 10,000 cells per capture) on primary breast tumors from 75 patients, representing all major PAM50 subtypes. For a subset of patients, we also collected matched stroma and/or NAT. As a control, we conducted LCM on non-tumor breast tissue from 42 healthy subjects (Table S2A). Global proteomic analysis was performed using liquid chromatography-tandem mass spectrometry (LC-MS/MS) (Table S2B). Notably, one of the most significantly upregulated proteins in NAT, and indeed one of the most NAT-specific proteins, compared to all other tissues examined was hepatocyte nuclear factor $4-\alpha(\mathrm{HNF} 4 \alpha)$ (Fig. 1F). As $\mathrm{HNF} 4 \alpha$ is an established, essential activator of lipolysis in adipose tissue (35), these data indicate lipolysis is highly activated in breast-tumor adjacent adipose tissue.

Fourth, we sought to validate the observations made in our clinical datasets using mouse models of breast cancer. Hormone sensitive lipase (HSL) is a critical lipolytic enzyme; its activation by protein kinase A (PKA) leads to phosphorylation at serine 563 (33), while prolonged activation results in down-regulation of total HSL expression through a negative feedback mechanism $(21,22)$. We performed immunoblot analysis to probe for HSL, phospho-HSL
(S563) and HNF4 $\alpha$ in tumor and NAT tissues from three wellcharacterized breast cancer patient-derived xenograft (PDX) models (HCI002, HCI009, HCI010) and a transgenic model of MYC-driven TNBC (MTBTOM) $(14,15)$, as well as corresponding control mammary tissues. In all models analyzed, total HSL was decreased in NAT compared to control tissues (Fig. $1, \mathrm{G}$ and $\mathrm{H})$. Downregulation of total HSL has been observed in obesity and in an independent analysis of primary breast tumor NAT, and is thought to be the result of a negative feedback loop in adipocytes in response to chronic lipolysis $(36,37)$. Additionally, in 3 of the 4 models examined we found an increase in HNF $4 \alpha$ or the phospho-HSL/HSL ratio (Fig. 1, G and H), both characteristic of increased lipolysis $(33,35)$. Taken together, our concurrent findings in 3 independent clinical datasets and several models of patient-derived and transgenic breast cancer in mice indicate that lipolysis is activated, albeit to varying degrees, in breast cancer-adjacent adipose tissue. These findings support the conclusion that "normal" tissue adjacent to tumors is in fact not normal (38); in the context of breast cancer, tumor-adjacent adipocytes have markers of activated lipolysis with corresponding diminished lipid stores.

We next sought to determine the contribution of cell-cell contact to lipolysis activation in breast tumor-adjacent adipocytes. Gap junctions are cell-cell junctions formed by a family of proteins called connexins, which are known to transport a variety of small molecules $(<1 \mathrm{kD})$, including cAMP $(34,39)$. Connexins were long thought to play tumor-suppressive roles in cancer, but recent evidence from a variety of tumor types has challenged this notion (39-42). Given that adipocytes are capable of transferring cAMP and activating lipolysis in a homotypic interaction (34), we hypothesized that gap junctions may form between tumor cells and adipocytes in a heterotypic fashion and activate lipolysis via transfer of cAMP. Using a well-established dye transfer assay (41), we first assayed for functional gap junction formation between breast cancer cells. We tested whether the TNBC cell line HCC1143 or the more indolent RP cell line T47D could transfer gap-junction dependent dyes to the same tumor cell line. Both lines formed functional gap junctions, but dye transfer amongst HCC1143 cells was 30-fold increased (Fig. 2A) compared to transfer amongst T47D cells. Thus, we reasoned there may be differences in sensitivity to gap junction inhibition between TN and RP cells. Furthermore, given the upregulation of the MYC oncogene in the majority of $\operatorname{TNBC}(43,44)$, we asked whether MYC expression affects gap junction dependence. We examined if gap junction inhibition alters cell viability in a panel of TN and RP human breast cell lines with varying MYC levels (24). We assayed intracellular ATP levels as a proxy for cell number. Intriguingly, TNBC cell lines with high MYC expression (24), including HCC1143, was significantly more sensitive to 48 hours of treatment with the pan-gap junction inhibitor carbenoxolone (CBX) than the low MYC TNBC or RP cell lines tested (Fig. 2B). These data suggest that gap junction communication occurs between breast cancer cells, and that a threshold amount of gap junction activity may be especially important in $\mathrm{TN}$ breast cancer cells with high MYC. 
To delineate the role of connexins in TN compared to RP breast cancer further, we examined the expression of the 21 connexin genes in 771 primary human $T N(n=123)$ and $R P(n=648)$ tumors using publically available RNAseq data from The Cancer Genome Atlas (TCGA). Of the 20 connexins for which data was available, 5/20 were significantly downregulated, 11/20 were significantly upregulated, and $4 / 20$ were not significantly changed in TN versus RP tumors (Fig. 2C). We noted that 5 of 7 members of the GJB class of gap junction proteins were upregulated (Fig. 2C). As an independent approach to examine expression of connexins in TNBC, we performed RNAseq on MTBTOM tumors and non-tumor control tissue (Table S3). Of the 10 connexins for which data were available, $2 / 10$ were significantly downregulated, 4/10 were significantly upregulated, and 4/10 were not significantly changed in MTBTOM tumors versus control tissue (Fig. 2D). Connexin 31 (GJB3, Cx31) was the most significantly elevated connexin in both human TN tumors and the MYC-driven TNBC model. Thus, we focused the remainder of our studies on $\mathrm{Cx} 31$. In non-tumor tissues $\mathrm{Cx} 31$ expression has been identified in keratinocytes, the small intestine, and the colon $(45,46)$. Although roles for various connexins as oncogenes and/or tumor suppressors have been described $(39,40)$, the function of $\mathrm{C} \times 31$ in tumorigenesis has not been established.

Accordingly, we sought to determine if functional Cx31containing gap junctions form between breast cancer cells and adipocytes. To model the direct cell-cell contact observed in vivo between breast cancer cells and adipocytes, we developed three independent co-culture approaches. First, we stably transduced HCC1143 (TNBC) and T47D (RP) cells with an mCherry expression plasmid. We then injected either mCherry-HCC1143 or -T47D cells directly into primary mammary adipose tissue isolated from a healthy individual (WD43177). After overnight co-culture we imaged the adipose tissue using confocal microscopy to identify regions of potential cell-cell contact. These regions were formalin-fixed and paraffin-embedded and probed for $\mathrm{Cx} 31$ and pan-cytokeratin expression, to distinguish epithelial tumor cells, using immunofluorescent microscopy. We found that HCC1143 cells formed close cell-cell contacts with primary adipocytes, and that both $\mathrm{HCC} 1143$ cells and adipocytes robustly expressed $\mathrm{Cx} 31$ at the plasma membrane (Fig. 3A). In contrast, T47D cells, despite forming cancer cell-cancer cell contacts, did not form close contacts with adipocytes (Fig. 3B). To further validate the presence of cancer-adipocyte gap junctions in TNBC, we examined $\mathrm{Cx} 31$ expression in primary patient biopsies. We found that both TN tumor cells and adipocytes robustly express $\mathrm{Cx} 31$ at the plasma membrane, and that many points of direct cellcell contact occur in vivo (Fig. 3C). These data suggest breast cancer cells are capable of forming close cell-cell contacts with adipocytes. Additionally, we note that our observations of such contacts correlated with the presence of $\mathrm{Cx} 31$ at both the tumor cell and adipocyte plasma membrane.

To determine whether breast cancer cells and adipocytes rely upon Cx31-dependent gap junctions, we utilized CRISPR/Cas9 technology to generate an allelic series of GJB3 knockout lines. Despite several attempts we were unable to generate any cancer cell lines with homozygous GJB3 deletion, strongly suggesting that a basal level of $\mathrm{Cx} 31$ expression is required for breast cancer cell growth. Therefore, we focused our studies on two TN lines, HS578T and HCC1143, that both have 3 copies of GJB3 to generate partial knockout lines. Specifically, we generated two independent clones of the HS578T line with 1 of 3 copies of GJB3 deleted (HS578T $1-\mathrm{GJB}^{+/+/}$and $2-\mathrm{GJB}^{+/+/}$), and from the HCC1143 line one clone with 1 of 3 copies deleted (HCC1143 $\mathrm{GJB}^{+/+/}$) and a second clone with 2 of 3 copies deleted $\left(\mathrm{HCC}_{1143 \mathrm{GJB}^{+/ / /}}\right.$) (Fig. 3D).

To determine if $\mathrm{Cx} 31$ expression impacted tumor cell-adipocyte communication we developed a co-culture model in which $\mathrm{HCC} 143 \mathrm{GJB}^{+/+/+}, \mathrm{GJB}^{+/+/-}$or GJB3 ${ }^{+/ /-}$cells were seeded in $2 \mathrm{D}$ culture and loaded with gap junction-transferable dye. We added primary mammary adipose tissue from three healthy individuals (WD42295, WD43911, WD50223) directly on top of the monolayers to ensure direct contact. Tumor cells and adipocytes were co-cultured for 5 hours and then assayed for gap junction-dependent dye transfer from the cancer cells to adipocytes. We found that robust dye transfer occurred from the HCC1143 GJB3 ${ }^{+/+/}$cells to mammary adipocytes of all three patients (Fig. 3E). However, reduction of $\mathrm{Cx} 31$ level by $1 / 3$ or $2 / 3$ in the $\mathrm{GJB}^{+/+/-}$and $\mathrm{GJB}^{+/ /-/}$lines, respectively, resulted in a significant decrease in dye transfer compared to $\mathrm{GJB}^{+/+/+}$control cells (Fig. 3E). These data suggest functional gap junctions form between TN breast cancer cells and adipocytes in a Cx31dependent manner.

Since adipocytes can activate lipolysis in neighboring adipocytes via gap junction-dependent transfer of cAMP (34), we suspected cAMP may be transferred via breast cancer cell-adipocyte gap junctions. To determine if breast cancer cell gap junctions are permeable to cAMP, we treated a panel of human TN and RP cell lines with CBX for 24 hours. In 5 of 6 lines tested we found marked increases in the levels of intracellular cAMP concentration in CBX-treated versus vehicle-treated cells (Fig. 3F). Additionally, significantly higher concentrations of cAMP were observed in high MYC TN cells in comparison to low MYC TN or RP cells (Fig. 3F). Given the recent observation that increased intracellular cAMP selectively decreases the viability of TN cells (47), our finding that high MYC TN cells display increased sensitivity to prolonged CBX treatment (Fig. 2B) may be due in part to increased levels of intracellular cAMP (Fig. 3F). Thus, the increase in intracellular cAMP upon pan-gap junction inhibition in 5 of 6 lines examined suggests that breast cancer cell gap junctions are indeed permeable to cAMP.

We next tested whether cAMP is directly transferred from breast cancer cells to adipocytes and if the abundance of $\mathrm{Cx} 31$ alters transfer. $\mathrm{HCC} 1143 \mathrm{GJB}^{+++/+}, \mathrm{GJB}^{+/+/-}$or $\mathrm{GJB}^{+/ /-}$cells were seeded and loaded with a fluorescent cAMP analogue (fluocAMP). These monolayer cultures were then co-cultured in direct contact with primary mammary adipose tissue from three healthy individuals (WD47558, WD46812, WD50344), and incubated for 5 hours. Adipocytes were then isolated from the tumor cells and assayed for cAMP. We found that cAMP transfer occurred from control cells to adipocytes from all three patients (Fig. 3G). However, as we observed with transfer of gap junction-permeable 
Camarda R, et al. 2018 - preprint version -www.biorxiv.org

dye (Fig. 3E), reduction of $\mathrm{Cx} 31$ expression resulted in a significant reduction of cAMP transfer (Fig. 3G). These results indicate that cAMP is transferred from TN breast cancer cells to adipocytes in a Cx31-dependent manner.

We next sought to determine if downstream cAMP signaling is activated in adipocytes in a tumor-adipocyte gap junctiondependent manner. To determine if cAMP signaling is activated in adipocytes upon cell-cell contact with breast cancer cells, we used a primary mouse preadipocyte model that can be differentiated to adipocytes in vitro (9). This model is ideal to study downstream signaling during co-culture because changes in adipocyte transcription can be assayed via qRT-PCR using murine-specific primers. Adipocytes were terminally differentiated and then HS578T and HCC1143 GJB3 partial knockout lines were seeded directly on top of adipocyte cultures. After co-culturing the cells for 24 hours we extracted RNA and assayed for changes in murine-specific (thus adipocyte-specific in this system) UCP1 expression, a known cAMP-responsive gene (9) to measure cAMP signaling. We also assayed for mouse aP2 expression as a marker of adipocyte differentiation. Our positive control, forskolin, which raises intracellular cAMP levels by activating adenylyl cyclase (9), robustly induced UCP1 expression compared to vehicle-treated cells (Fig. $3 \mathrm{H}$ ). The $\mathrm{HCC}_{1143 \mathrm{GJB}^{+/+++}}$and $\mathrm{GJB}^{+/+/}$lines both induced UCP1 expression, but UCP1 induction was significantly reduced with the GJB3 ${ }^{+/-/}$cells (Fig. $3 \mathrm{H}$ ). In contrast, none of the HS578T lines, including the $\mathrm{GJB}^{+/+++}$control, were capable of inducing UCP1 expression (Fig. 3H). All conditions, including forskolin treatment, resulted in reduced aP2 expression (Fig. 3H), suggesting effects on adipocyte differentiation are distinct from those observed on cAMP signaling. Given that $\mathrm{Cx} 31$ expression is similar in HS578T GJB3 $3^{+/++}$and $\mathrm{HCC} 1143 \mathrm{GJB}^{+/ / /}$cells (Fig. 3D), and that neither activate cAMP signaling (Fig. $3 \mathrm{H}$ ), it is possible that a $\mathrm{Cx} 31$ expression threshold is required for breast cancer cells to activate cAMP signaling in adjacent adipocytes. Although direct transfer of cAMP has been described between adipocytes in a homotypic interaction (34), here we identify for the first time a gap junction-dependent activation of lipolysis in adipocytes by tumor cells.

Finally, we sought to determine the contribution of breast cancer Cx31-dependent gap junctions to tumorigenesis. We found that HS578T 1-GJB3 $3^{++/-}$and 2-GJB3 $3^{++/}$, and HCC1143 $\mathrm{GJB}^{+++/}$ lines did not display a difference in proliferation compared to their respective $\mathrm{GJB}^{+++/+}$control lines. In contrast, $\mathrm{HCC} 1143 \mathrm{GJB}^{+/-}$ 1- cells demonstrate a significant reduction in cell proliferation (Fig. 4A), but no reduction in cell viability (data not shown). These data suggest that, even in the absence of breast cancer celladipocyte interaction, Cx31 may promote breast cancer cell proliferation. To determine the contribution of $\mathrm{Cx} 31$ to breast tumorigenesis in vivo, we transplanted each of the HS578T and HCC1143 Cx31 partial knockout lines into cleared mammary fat pads of immunocompromised NOD-SCID/gamma (NSG) female mice and determined the time of tumor onset and time to reach ethical endpoint (when tumor reaches $2 \mathrm{~cm}$ in any dimension). Remarkably, with the HS578T lines, in which partial GJB3 knockout had no effect on cell proliferation in vitro (Fig. 4A),
0/10 mice that received HS578T $1-\mathrm{GJB}^{+/+/-}$or $2-\mathrm{GJB}^{+/+/-}$ xenografts ( 5 per line) developed tumors within 180 days (Fig. 4B). Among the HCC1143 lines, the GJB3 $3^{++/-}$line displayed a significant delay in both tumor onset and time to ethical endpoint, while only 3 of 5 mice transplanted with the $\mathrm{GJB}^{+/-/}$line developed tumors, and none reached ethical endpoint within 180 days (Fig. 4B). Our data indicates that $\mathrm{Cx} 31$ promotes breast tumorigenesis in vivo.

We sought to clarify the effects of Cx31 expression on lipolysis versus other effects on tumor growth. To determine if control and Cx31 partial knockout tumors differentially induced lipolysis, we collected tumor and NAT from $\mathrm{HCC} 1143 \mathrm{GJB}^{+/+/+}, \mathrm{GJB}^{+/+/-}$and $\mathrm{GJB}^{+-/-l}$ tumor-bearing mice, as well as residual mammary glands from the two $\mathrm{GJB}^{+/-/}$mice that were transplanted, but never developed tumors. Using immunoblot analysis, we probed for markers of lipolysis. Notably, a marked reduction in total HSL expression was found in 3 of $3 \mathrm{HCC} 1143 \mathrm{GJB}^{+/+/+} \mathrm{NAT}$ samples compared to control tissues, consistent with persistent activation of lipolysis (Fig. 4C). In contrast, we did not observe a consistent change in HSL expression in any of the other NAT samples analyzed from tumors with partial Cx31 knockout (Fig. 4C). Interestingly, we found a marked increase in phospho-HSL/HSL ratio in both the $\mathrm{HCC} 1143 \mathrm{GJB}^{+/+/+}$and $\mathrm{GJB}^{+/+/-} \mathrm{NAT}$ samples, but this difference was significantly reduced in $\mathrm{HCC} 1143 \mathrm{GJB}^{+/-}$ 1- NAT (Fig. 4C). The increase in phospho-HSL/HSL in GJB3 ${ }^{+/ /-}$ NAT may be due to alternative modes of lipolysis activation, such as secreted pro-lipolytic cytokines (26), which is congruent with the observed increase in UCP1 expression during $\mathrm{GJB}^{+/+/}$adipocyte co-culture (Fig. 3H). To further interrogate lipolytic signaling in NAT, we probed for cAMP abundance in HCC1143 $\mathrm{GJB}^{+/+++}$and $\mathrm{GJB}^{+/+/-}$tumors by mass spectrometry. We found a significant increase in intratumoral cAMP level in HCC1143 $\mathrm{GJB}^{+/+/}$tumors compared to the $\mathrm{GJB}^{+++/+}$control tumors (Fig. $4 \mathrm{D})$, consistent with diminished transfer of cAMP to NAT. We examined $\mathrm{GJB}^{+/+/+}$and $\mathrm{GJB}^{+/+/}$tumors and associated NAT, and assayed for differences in adjacent adipocyte size, as an indicator of lipolysis. Strikingly, we found a significant increase in the average size of adipocytes adjacent to $\mathrm{GJB}^{+++/}$tumors compared to $\mathrm{GJB}^{+/+++}$control tumors (Fig. 4E), again supporting a decreased induction of lipolysis in NAT from Cx31 partial knockout tumors. Finally, if the delay in $\mathrm{HCC} 1143 \mathrm{GJB}^{+/+/}$ tumor onset (Fig. 4B) was due to an inability to activate lipolysis in adjacent adipocytes, we reasoned that pharmacological activation of lipolysis should rescue this phenotype. Indeed, we found that daily intra-peritoneal injection of CL316273, a specific $\beta 3$-receptor agonist known to activate lipolysis in vivo (48), completely rescued the delay in tumor onset observed in $\mathrm{HCC} 1143 \mathrm{GJB}^{+++/}$tumors, but did not further promote the growth of $\mathrm{HCC} 1143 \mathrm{GJB}^{+/+/+}$tumors (Fig. 4F). Taken together, these data indicate that cAMP signaling and lipolysis are activated in breast tumor-adjacent adipocytes in a Cx31-dependent manner in vivo.

In summary, we find that lipolysis is activated in breast canceradjacent adipose tissue and that functional gap junctions form between breast cancer cells, and between breast cancer cells and adipocytes. In addition, cAMP is transferred via breast cancer cell 
Camarda R, et al. 2018 - preprint version -www.biorxiv.org

gap junctions, and cAMP signaling is activated in adipocytes adjacent to breast cancer cells in a gap junction-dependent manner. Finally, we discovered a previously unappreciated role for Cx31-dependent gap junctions in breast tumor growth and activation of lipolysis in tumor-adjacent adipose tissue in vivo. Cx31 may represent a new therapeutic target to treat pro-lipolytic breast tumors. Furthermore, the recent discovery of gap junction formation between brain metastatic carcinoma cells and astrocytes (41) suggests that gap junction-dependent heterotypic interaction between tumor and non-tumor cells may be an emerging hallmark of tumorigenesis.

\section{Conflict of interest:}

The authors declare no conflicts of interest.

\section{Acknowledgements:}

This work was supported, in part, by the US Department of Defense-Congressionally Directed Medical Research Programs' Era of Hope Scholar award W81XWH-12-1-0272 (A.G.), the US National Institutes of Health (NIH) grants R01 CA170447 (A.G.), R01 CA166945 (J.A.S.), and R01 CA056721 (Z.W.), the Atwater Foundation (A.G.), the California Breast Cancer Research Program grant CBCRP 18IB-0042 (J.A.S.), the Breast Cancer Research Fund (H.R.), the EMBO postdoctoral fellowship ALTF 159-2017 (J.W.) and the US NIH F99/K00 Predoctoral to Postdoctoral Transition Award F99CA212488 (R.C.). The authors thank A. Welm for guidance in the use of patient-derived xenografts, A. Tward for technical guidance and helpful discussions, K.A. Fontaine for helpful discussions and comments on the manuscript, and S. Samson for a helpful consumer advocate perspective and guidance on our work. R.C. and A.G. conceived of research. R.C. designed and contributed to all in vitro studies and the in vivo mouse studies, contributed to all data analysis, and wrote the manuscript. J.W. designed and contributed to in vitro studies. S.M. analyzed 3CB data. L.J.Z. designed LCM and conducted proteomic analyses. S.M. conducted the LCM. D.A. conducted lipolysis signature enrichment analysis. A.B. contributed to immunofluorescent staining and microscopy, and generation of $\mathrm{Cx} 31$ partial knockout lines. D.V.d.M. contributed to the CL316273 mouse study. Y.C. conducted preadipocyte differentiation. C.B. and S.L. conducted mass spectrometry for cAMP. C.M. generated Cas9-expressing cancer cell lines. J.W. and E.W. isolated primary mammary adipose tissue. J.D.G. conducted FACS analysis. K.S. provided cAMP-dependent lipolysis signature. K.M.A. supervised FACS analysis. Z.W. supervised primary mammary adipose tissue isolation and provided valuable discussion. D.K.N. supervised mass spectrometry for cAMP and provided valuable discussion. S.K. supervised preadipocyte and cAMP-dependent lipolysis studies and provided valuable discussion. A.J.B supervised enrichment analysis. M.E.S. and D.C.L. designed and supervised LCM and proteomics. H.R. provided valuable discussion. G.K. designed and conducted histological analyses and provided valuable discussion. J.A.S. supervised the $3 \mathrm{CB}$ study and data analysis and provided valuable discussion. A.G. supervised the study and provided valuable discussion and intellectual input. All authors edited the manuscript. All data and code related to these studies are available in the main text, supplementary materials and indicated repositories. The raw RNAseq data will be deposited on GEO. The authors declare no competing interest.

\section{References:}

1. A. D. Laidevant, S. Malkov, C. I. Flowers, K. Kerlikowske, J. A. Shepherd, Compositional breast imaging using a dual-energy mammography protocol. Med. Phys. 37, 164-174 (2009).

2. S. Malkov, J. Wang, K. Kerlikowske, S. R. Cummings, J. A. Shepherd, Single x-ray absorptiometry method for the quantitative mammographic measure of fibroglandular tissue volume. Med. Phys. 36, 5525-5536 (2009).

3. S. Malkov, J. Wang, F. Duewer, J. A. Shepherd, in International Workshop on Digital Mammography (2012; http://link.springer.com/10.1007/978-3-642-312717_99), pp. 769-774.

4. S. Malkov et al., in International Workshop on Digital Mammography http://link.springer.com/10.1007/978-3-319-41546-8), vol. 9699 , pp. 211-218.

5. K. Drukker et al., Mammographic quantitative image analysis and biologic image composition for breast lesion characterization and classification. Med. Phys. 41, 31915 (2014).

6. M. E. H. Hammond et al., American Society of Clinical Oncology/College of American Pathologists guideline recommendations for immunohistochemical testing of estrogen and progesterone receptors in breast cancer (unabridged version). Arch. Pathol. Lab. Med. 134, e4872 (2010).

7. A. C. Wolff et al., Recommendations for Human Epidermal Growth Factor Receptor 2 Testing in Breast Cancer: American Society of Clinical Oncology/College of American Pathologists Clinical Practice Guideline Update. J. Clin. Oncol. 31, 3997-4013 (2013).

8. M. Galarraga et al., Adiposoft: automated software for the analysis of white adipose tissue cellularity in histological sections. J. Lipid Res. 53, 2791-2796 (2012).

9. K. Shinoda et al., Genetic and functional characterization of clonally derived adult human brown adipocytes. Nat. Med. 21, 389-394 (2015).

10. R. G. W. Verhaak et al., Prognostically relevant gene signatures of high-grade serous ovarian carcinoma. $J$. Clin. Invest. 123, 517-25 (2013).

11. A. S. L. Cheng et al., Epithelial progeny of estrogenexposed breast progenitor cells display a cancer-like methylome. Cancer Res. 68, 1786-96 (2008).

12. M. Dai et al., Evolving gene/transcript definitions significantly alter the interpretation of GeneChip data. Nucleic Acids Res. 33 (2005), doi:10.1093/nar/gni179.

13. B. Zhang et al., Proteogenomic characterization of human colon and rectal cancer. Nature. 513, 382-387 (2014).

14. Y. S. DeRose et al., Tumor grafts derived from women with breast cancer authentically reflect tumor pathology, growth, metastasis and disease outcomes. Nat. Med. 17 (2011), pp. 1514-1520.

15. C. M. D'Cruz et al., c-MYC induces mammary 
Camarda R, et al. 2018 - preprint version -www.biorxiv.org

tumorigenesis by means of a preferred pathway involving spontaneous Kras2 mutations. Nat. Med. 7, 235-239 (2001).

16. R. M. Neve et al., A collection of breast cancer cell lines for the study of functionally distinct cancer subtypes. Cancer Cell. 10, 515-527 (2006).

17. G. K. Smyth, limma: Linear Models for Microarray Data. Bioinforma. Comput. Biol. Solut. Using R Bioconductor, 397-420 (2005).

18. A. Ettinger, T. Wittmann, Fluorescence live cell imaging (Elsevier Inc., ed. 1, 2014; http://dx.doi.org/10.1016/B978-0-12-420138-5.00005-

7), vol. 123.

19. S. Stehbens, H. Pemble, L. Murrow, T. Wittmann, Imaging intracellular protein dynamics by spinning disk confocal microscopy (Elsevier Inc., ed. 1, 2012; http://dx.doi.org/10.1016/B978-0-12-391857-4.00015X), vol. 504.

20. S. J. Stehbens et al., CLASPs link focal-adhesionassociated microtubule capture to localized exocytosis and adhesion site turnover. Nat. Cell Biol. 16, 558-570 (2014).

21. S. Kajimura et al., Initiation of myoblast to brown fat switch by a PRDM16-C/EBP- $\beta$ transcriptional complex. Nature. 460, 1154-1158 (2009).

22. K. J. Livak, T. D. Schmittgen, Analysis of Relative Gene Expression Data Using Real-Time Quantitative PCR and the 2- $\Delta \Delta$ CT Method. Methods. 25, 402-408 (2001).

23. P. J. Goodwin, R. T. Chlebowski, Obesity and cancer: Insights for clinicians. J. Clin. Oncol. 34, 4197-4202 (2016).

24. R. Camarda et al., Inhibition of fatty acid oxidation as a therapy for MYC-overexpressing triple-negative breast cancer. Nat. Med. 22, 427-432 (2016).

25. J. H. Park et al., Fatty Acid Oxidation-Driven Src Links Mitochondrial Energy Reprogramming and Oncogenic Properties in Triple-Negative Breast Cancer. Cell Rep. 14, 2154-65 (2016).

26. H. Ye et al., Leukemic Stem Cells Evade Chemotherapy by Metabolic Adaptation to an Adipose Tissue Niche. Cell Stem Cell. 19, 23-37 (2016).

27. K. M. Nieman et al., Adipocytes promote ovarian cancer metastasis and provide energy for rapid tumor growth. Nat. Med. 17, 1498-1503 (2011).

28. S. Balaban et al., Adipocyte lipolysis links obesity to breast cancer growth: adipocyte-derived fatty acids drive breast cancer cell proliferation and migration. Cancer Metab. 5, 1 (2017).

29. Y. Y. Wang et al., Mammary adipocytes stimulate breast cancer invasion through metabolic remodeling of tumor cells. JCI Insight. 2 (2017), doi:10.1172/jci.insight.87489.

30. Y.-A. Wen et al., Adipocytes activate mitochondrial fatty acid oxidation and autophagy to promote tumor growth in colon cancer. Cell Death Dis. 8, e2593 (2017).

31. A. J. Hoy, S. Balaban, D. N. Saunders, Adipocyte-Tumor Cell Metabolic Crosstalk in Breast Cancer. Trends Mol. Med. 23, 381-392 (2017).
32. R. C. Hovey, L. Aimo, Diverse and active roles for adipocytes during mammary gland growth and function. J. Mammary Gland Biol. Neoplasia. 15, 279-290 (2010).

33. R. E. Duncan, M. Ahmadian, K. Jaworski, E. SarkadiNagy, H. S. Sul, Regulation of Lipolysis in Adipocytes. Annu. Rev. Nutr. 27, 79-101 (2007).

34. Y. Zhu et al., Connexin 43 Mediates White Adipose Tissue Beiging by Facilitating the Propagation of Sympathetic Neuronal Signals. Cell Metab. 24, 420-433 (2016).

35. L. Palanker, J. M. Tennessen, G. Lam, C. S. Thummel, Drosophila HNF4 Regulates Lipid Mobilization and $\beta$ Oxidation. Cell Metab. 9, 228-239 (2009).

36. V. Large et al., Decreased expression and function of adipocyte hormone-sensitive lipase in subcutaneous fat cells of obese subjects 1. J. Lipid Res. 40, 2059-2066 (1999).

37. F. Wang et al., Mammary fat of breast cancer: Gene expression profiling and functional characterization. PLoS One. 9 (2014), doi:10.1371/journal.pone.0109742.

38. D. Aran et al., Comprehensive analysis of normal adjacent to tumor transcriptomes. Nat. Commun. 8, 1077 (2017).

39. T. Aasen, M. Mesnil, C. C. Naus, P. D. Lampe, D. W. Laird, Gap junctions and cancer: communicating for 50 years. Nat. Rev. Cancer. 16, 775-788 (2016).

40. M. A. Gleisner, M. Navarrete, F. Hofmann, F. SalazarOnfray, A. Tittarelli, Mind the Gaps in Tumor Immunity: Impact of Connexin-Mediated Intercellular Connections. Front. Immunol. 8, 1-17 (2017).

41. Q. Chen et al., Carcinoma-astrocyte gap junctions promote brain metastasis by cGAMP transfer. Nature. 533, 493-498 (2016).

42. M. Osswald et al., Brain tumour cells interconnect to a functional and resistant network. Nature. 528, 93-98 (2015).

43. D. Horiuchi et al., MYC pathway activation in triplenegative breast cancer is synthetic lethal with CDK inhibition. J. Exp. Med. 209, 679-696 (2012).

44. D. C. Koboldt et al., Comprehensive molecular portraits of human breast tumours. Nature. 490, 61-70 (2012).

45. K. Wenzel, D. Manthey, K. Willecke, K. H. Grzeschik, O. Traub, Human gap junction protein connexin31: molecular cloning and expression analysis. Biochem Biophys Res Commun. 248, 910-915 (1998).

46. J. Willebrords, M. Maes, S. Crespo Yanguas, M. Vinken, Inhibitors of connexin and pannexin channels as potential therapeutics. Pharmacol. Ther. (2017), , doi:10.1016/j.pharmthera.2017.07.001.

47. W. Wang et al., Triple negative breast cancer development can be selectively suppressed by sustaining an elevated level of cellular cyclic AMP through simultaneously blocking its efflux and decomposition. Oncotarget (2016), doi:10.18632/oncotarget.13601.

48. Q. A. Wang, C. Tao, R. K. Gupta, P. E. Scherer, Tracking adipogenesis during white adipose tissue development, expansion and regeneration. Nat. Med. 19, 1338-1344 (2013). 


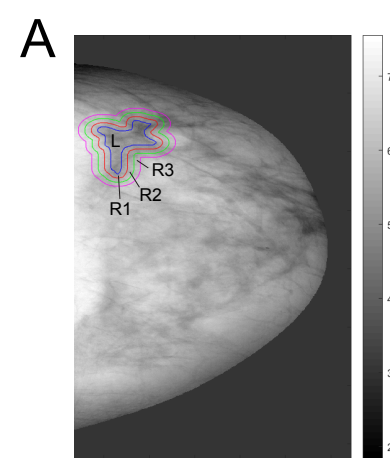

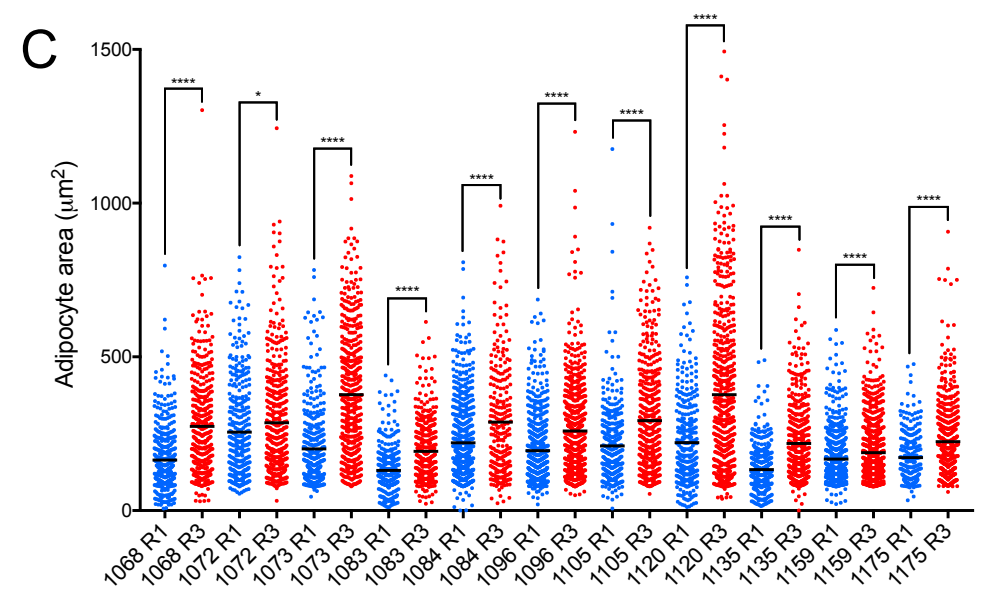
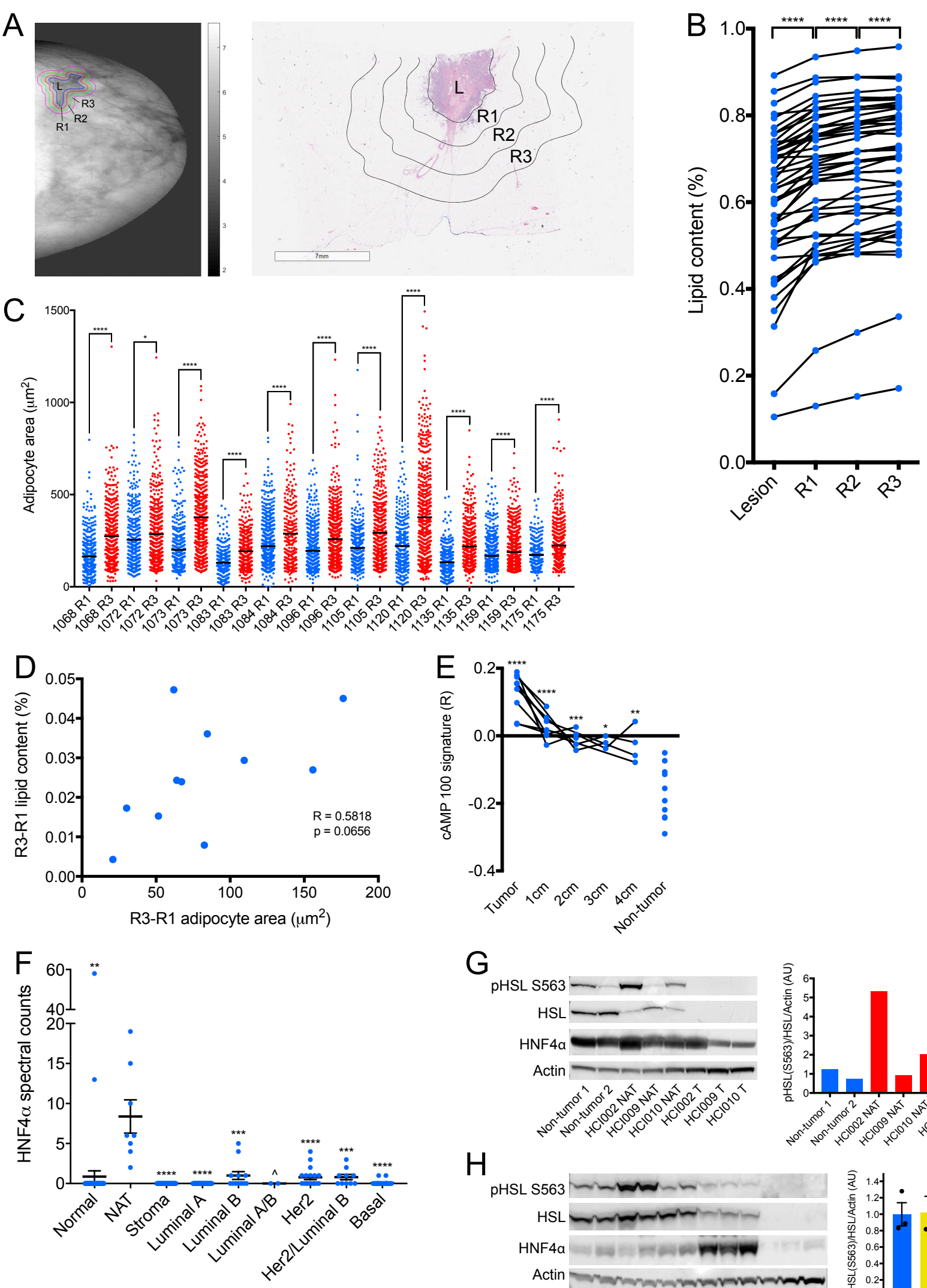

Figure 1

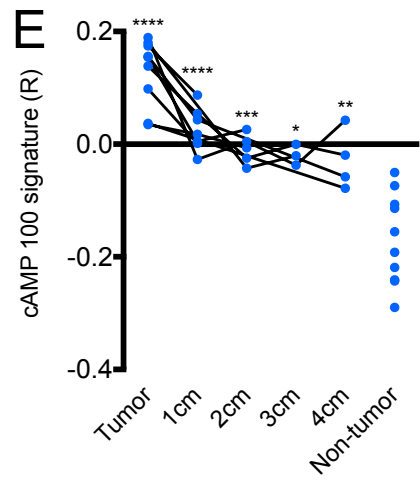

G

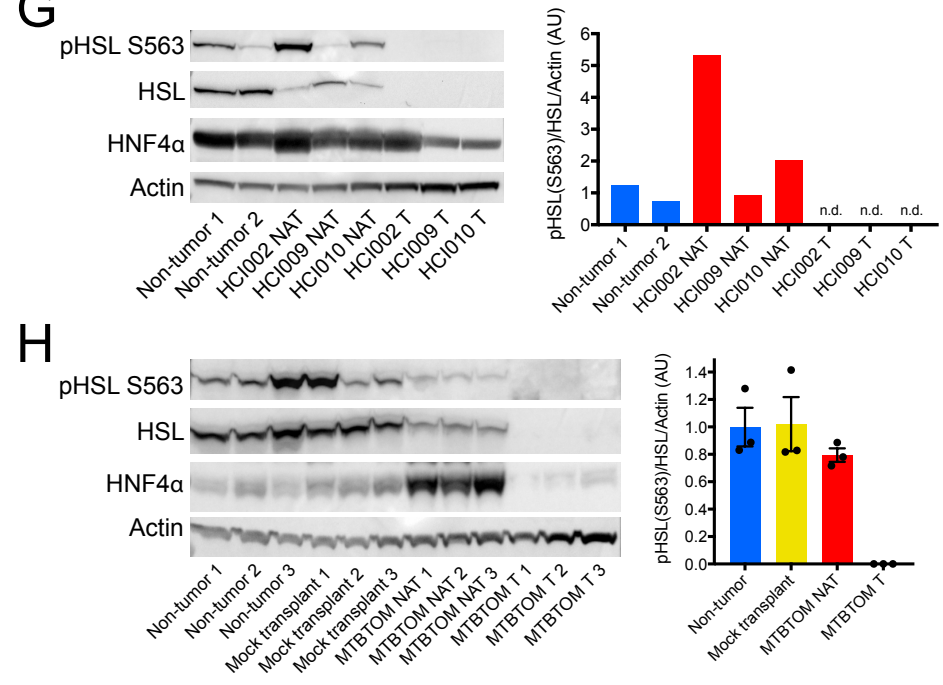


Camarda R, et al. 2018 - preprint version -www.biorxiv.org

Fig. 1. Lipolysis and lipolytic signaling are activated in breast tumor-adjacent adipocytes from breast cancer patients and mouse models of breast cancer. (A) Representative lipid content image (left) and hematoxylin and eosin stained excision specimen (right) from patients with invasive breast cancer. The lesion (L), and NAT 0-2 mm (R1), 2-4 mm (R2), and 4-6 mm (R3) away are indicated. (B) Percent lipid content (lipid content / lipid + water + protein content) of L, R1, R2 and R3 from patients $(n=46)$ with invasive breast cancer. $(C)$ Adipocyte area in R1 and R3 from a subset of patients $(\mathrm{n}=11)$ in B. The black line indicates mean adipocyte area, and each patient identifier is indicated. Each point represents individual adipocyte. (D) Correlation of change in lipid content in B and change in average adipocyte area in $\mathrm{C}$ from R3 to R1 for patients in C. Spearman correlation and two-tailed t test were used to generate the correlation coefficient and associated $\mathrm{P}$ value. (E) ssGSEA enrichment scores for cAMP-dependent lipolysis signature in primary breast tumors (n $=9)$, NAT $1 \mathrm{~cm}(\mathrm{n}=7), 2 \mathrm{~cm}(\mathrm{n}=5), 3 \mathrm{~cm}(\mathrm{n}=3)$, and $4 \mathrm{~cm}(\mathrm{n}=4)$, and healthy non-tumor breast tissue $(\mathrm{n}=10)$. (F) HNF4 $\alpha$ peptide counts from LC-MS/MS of primary tissue from healthy control breast tissue $(n=42)$, NAT $(n=4)$, stroma ( $n=36)$, and luminal A ( $n$ = 38), luminal B $(\mathrm{n}=6)$, luminal A/B $(\mathrm{n}=1)$, HER2-amplified $(\mathrm{n}=9)$, HER2-amplified/luminal B $(\mathrm{n}=5)$, and basal $(\mathrm{n}=16)$ tumors. Each point represents individual sample LCM on which LC-MS/MS was performed. LCM and LC-MS/MS was performed in technical duplicate on sequential histological slides from each patient. (G) Immunoblot analysis (left) showing expression levels of lipolysis activators HSL and HNF4 $\alpha$, and phosphorylated HSL (pHSL S563) in healthy non-tumor mammary gland and NAT and tumor tissues from a panel of PDXs. Quantification (right) of pHSL/HSL ratio, normalized to $\beta$-actin levels. (H) Immunoblot analysis (left) showing expression levels of lipolysis activators HSL and HNF4 $\alpha$, and phosphorylated HSL (pHSL S563) in healthy non-tumor mammary gland, mock-transplanted mammary gland, and NAT and tumor tissues from MTBTOM allografts. Quantification (right) of pHSL/HSL ratio, normalized to $\beta$-actin levels. For $(\mathrm{B})$ and $(\mathrm{E})$ black lines indicate matched samples from individual patients. For $(\mathrm{F})$ and $(\mathrm{H})$ mean \pm s.e.m. is shown. ${ }^{\wedge} \mathrm{P}<0.10,{ }^{*} \mathrm{P}<0.05, * * \mathrm{P}<0.01, * * * \mathrm{P}<0.001$, $* * * * \mathrm{P}<0.0001$; paired two-tailed t test (B), unpaired two-tailed t test $(\mathrm{C}),(\mathrm{E})$, and $(\mathrm{F})$. 


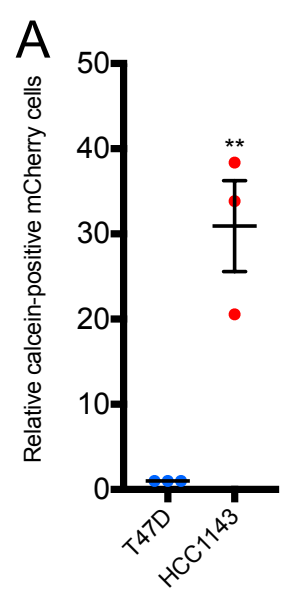

B
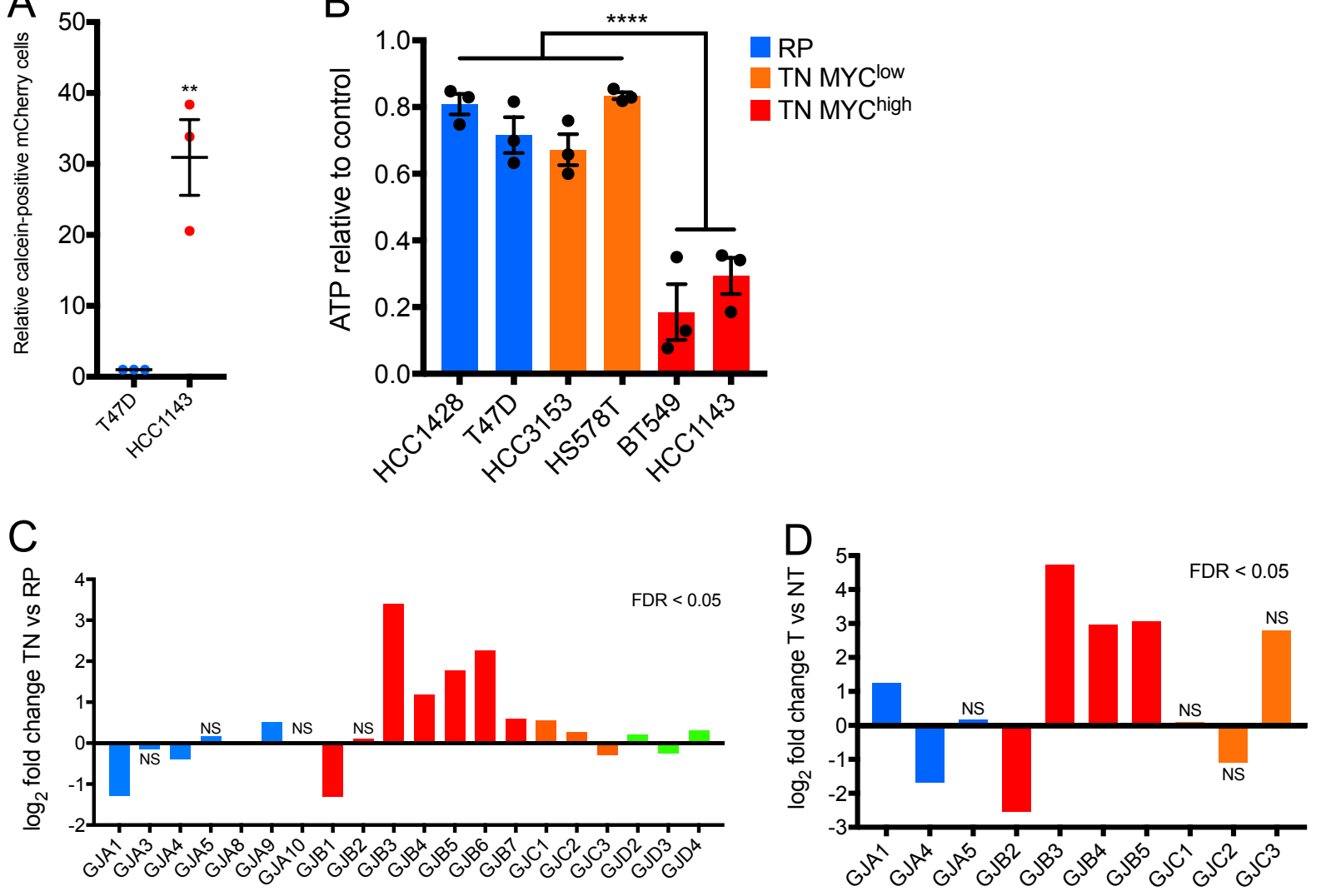

Figure 2 
Camarda R, et al. 2018 - preprint version -www.biorxiv.org

Fig. 2. Breast cancer cells form functional gap junctions and express Cx31. (A) Relative frequency of dye transfer from Calcein AMloaded cells (donor) to mCherry-labelled cells (recipient) as determined by FACS (fluorescence-activated cell sorting) analysis. (B) ATP levels in TN high MYC, TN low MYC, and RP cell lines after treatment with $150 \mu \mathrm{M} \mathrm{CBX}$ for 48 hours relative to untreated (control) cells. (C) Fold change $\left(\log _{2}\right)$ in expression of indicated connexin genes in TN $(n=123)$ versus RP $(n=648)$ tumors based on RNA-seq data acquired from TCGA of 771 breast cancer patients. (D) Fold change $\left(\log _{2}\right)$ in expression of indicated connexin genes in $\mathrm{T}(\mathrm{n}=11)$ versus NT $(n=3)$ tissues based on RNA-seq data from MTBTOM allograft-bearing mice or healthy controls, respectively. For (A) and (B) mean \pm s.e.m. of three independent biological replicates is shown. ${ }^{* * \mathrm{P}}<0.01$, ${ }^{* * * * \mathrm{P}}<0.0001$; unpaired two-tailed t test (A) and (B). For (C) and (D) all differential expression analysis was done using the 'limma' R package. 

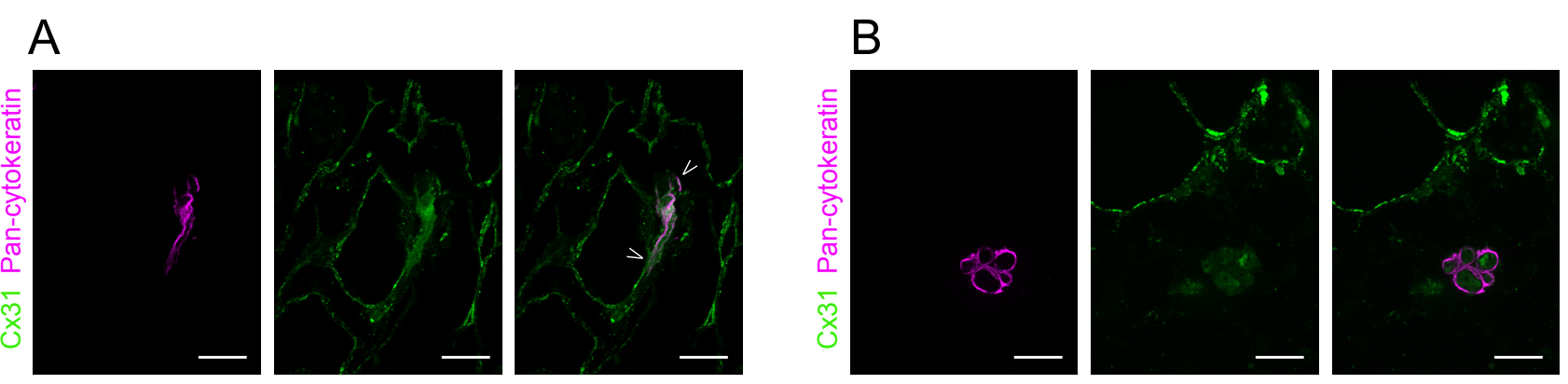

\section{C}
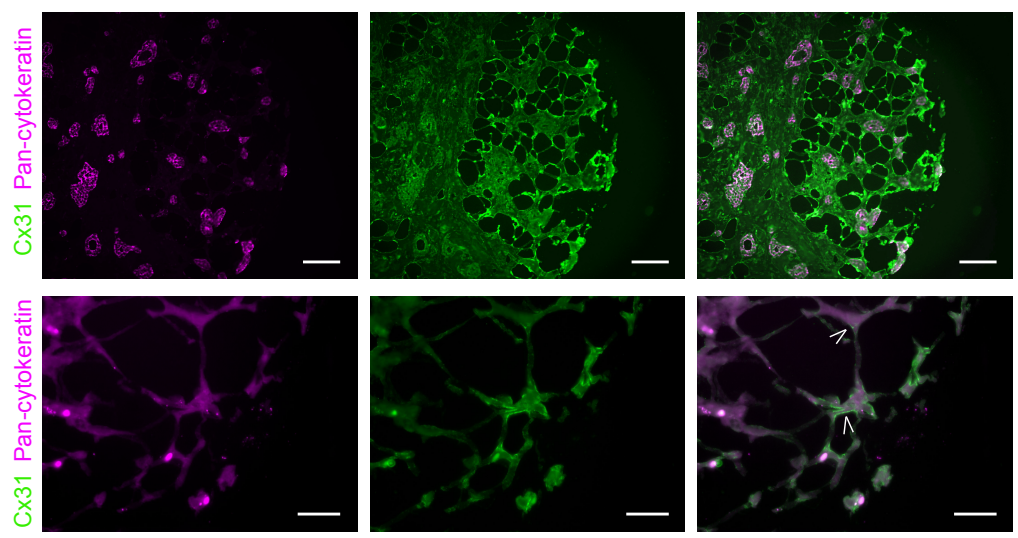

D
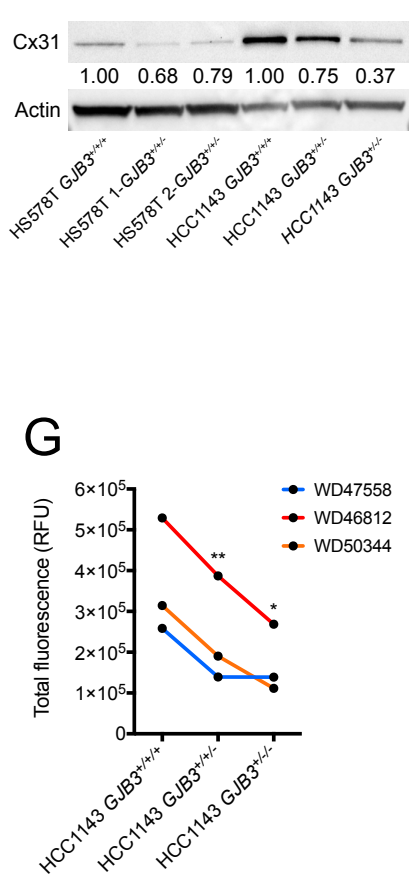
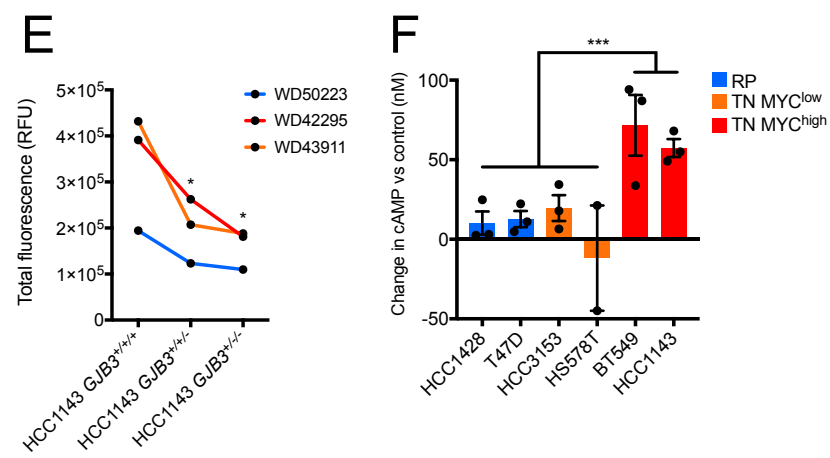

Figure 3

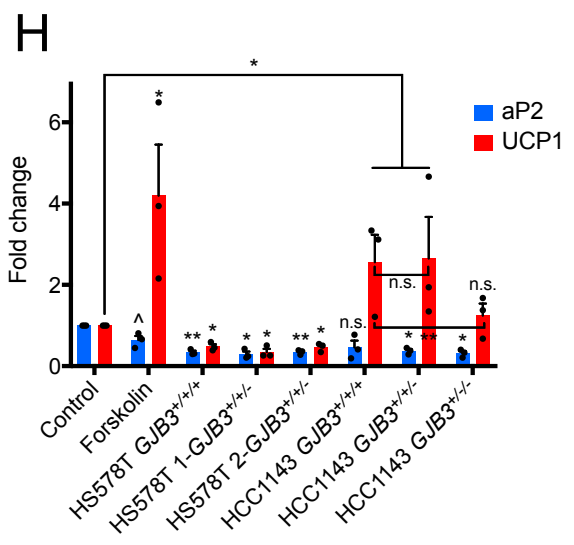


Camarda R, et al. 2018 - preprint version -www.biorxiv.org

Fig. 3. Breast cancer cell-adipocyte gap junctions form, transfer cAMP, and activate lipolytic signaling in a Cx31-dependent manner. (A) Staining with Cx31 (green) and pan-cytokeratin (magenta) of primary mammary tissue from a healthy individual (WD43177) injected with TN mCherry-HCC1143 cells and co-cultured overnight. White arrowheads indicate co-staining of Cx31 with contact point HCC1143 and adipocyte plasma membranes. Scale bar, $25 \mu \mathrm{m}$. (B) Staining with Cx31 (green) and pan-cytokeratin (magenta) of primary mammary adipose tissue from a healthy individual (WD43177) injected with RP mCherry-T47D cells and co-cultured overnight. Scale bar, $25 \mu \mathrm{m}$. (C) Staining with Cx31 (green) and pan-cytokeratin (magenta) of primary TNBC patient biopsies. Scale bar, top $100 \mu \mathrm{m}$, bottom $25 \mu \mathrm{m}$. (D) Immunoblot analysis showing expression levels of Cx31 in a panel of clonally derived control $\left(\mathrm{GJB}^{+/++}\right)$and $\mathrm{Cx} 31$ partial knockdown TN lines. For the Cx31-knockdown lines each clone is referred to by number of GJB3 alleles expressed (e.g. $\mathrm{GJB}^{+++/}$is missing $1 / 3$ functional alleles). Quantification of $\mathrm{Cx} 31$ level normalized to $\beta$-actin level is indicated. (E) Dye transfer from indicated $\mathrm{HCC} 1143$ control and $\mathrm{Cx} 31$ partial knockout lines to primary mammary adipose tissue of indicated healthy individuals. (F) cAMP levels in TN high MYC, TN low MYC, and RP cell lines after treatment with $150 \mu \mathrm{M} \mathrm{CBX}$ for 24 hours relative to untreated (control) cells. (G) cAMP transfer from indicated HCC1143 control and Cx31-knockdown lines to primary mammary adipose tissue of indicated healthy individuals. (H) Fold change in UCP1 and aP2 expression in differentiated adipocytes after treatment with vehicle (control) or $10 \mu \mathrm{M}$ forskolin, or co-cultured with indicated $\mathrm{Cx} 31$ partial knockout lines for 24 hours. For $(\mathrm{F})$ and $(\mathrm{H})$ mean \pm s.e.m. of three independent biological replicates is shown. ${ }^{\wedge} \mathrm{P}<0.10,{ }^{*} \mathrm{P}<0.05,{ }^{*} \mathrm{P}<0.01,{ }^{* * *} \mathrm{P}<0.001$; paired two-tailed $\mathrm{t}$ test $(\mathrm{E})$, $(\mathrm{G})$, and $(\mathrm{H})$, unpaired two-tailed $\mathrm{t}$ test $(\mathrm{F})$. 

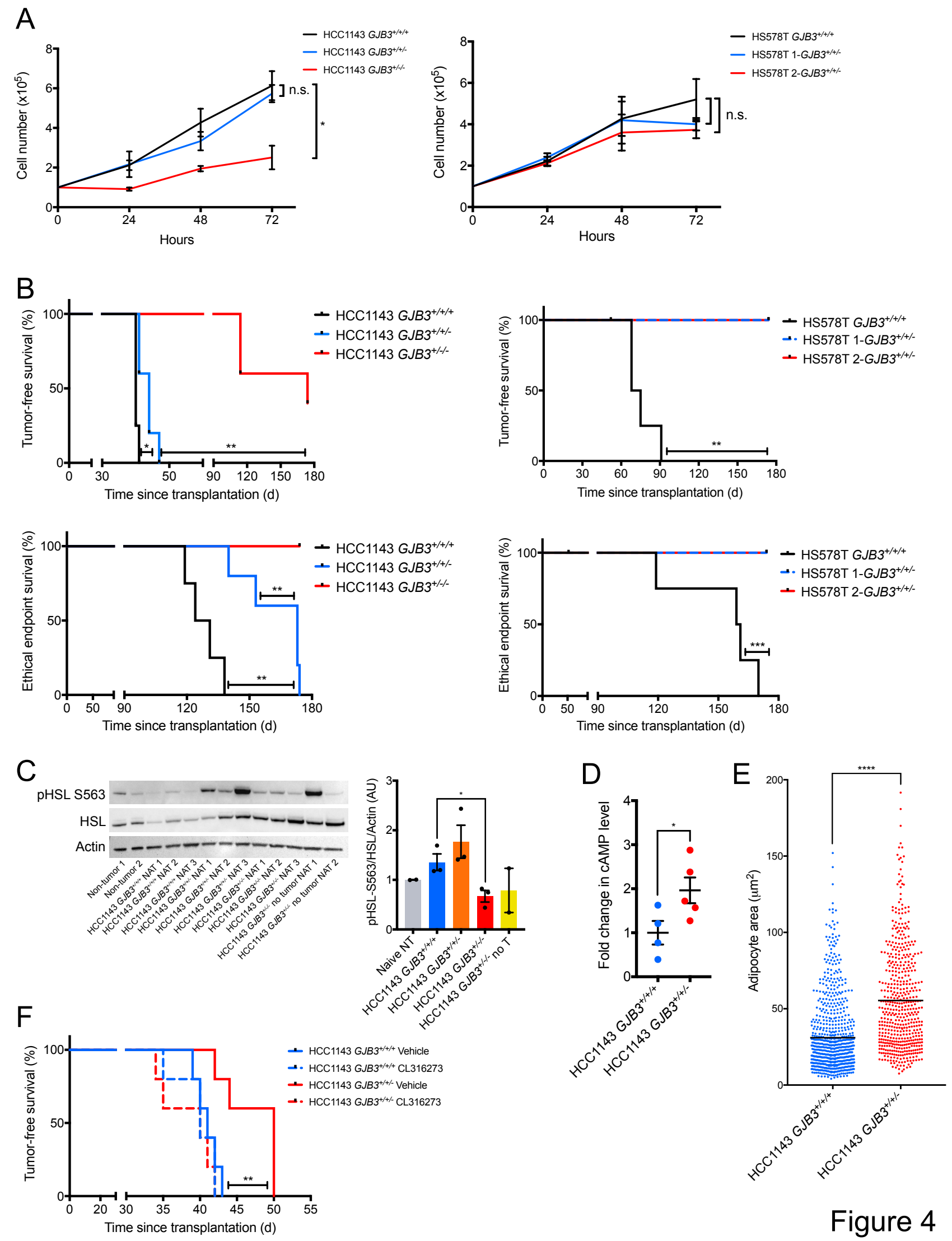

Figure 4 
Camarda R, et al. 2018 - preprint version -www.biorxiv.org

Fig. 4. $\mathrm{Cx} 31$ is essential for breast cancer cell growth in vitro, and tumorigenesis and activation of lipolysis in adjacent adipocytes in vivo. (A) Cell growth of indicated Cx31 partial knockout lines over 72 hours. (B) Kaplan-Meier analysis of tumor onset (top) and ethical endpoint survival (bottom) of mice bearing indicated Cx31 partial knockout orthotopic xenografts ( $\mathrm{n}=5$ per group). Ethical endpoint survival indicates the percentage of mice bearing xenografts $<2 \mathrm{~cm}$ in any dimension. (C) Immunoblot analysis (left) showing expression levels of HSL and phosphorylated HSL (pHSL S563) in healthy non-tumor mammary gland and NAT from mice bearing indicated Cx31 partial knockout xenografts or mice that were transplanted, but subsequently did not develop a tumor. Quantification (right) of pHSL/HSL ratio, normalized to $\beta$-actin levels. (D) Fold change in cAMP levels in HCC1143 $\mathrm{GJB}^{+/+/}$xenografts versus $\mathrm{HCC} 1143$ $\mathrm{GJB}^{+++/+}$xenografts. (E) Adipocyte area adjacent to $\mathrm{HCC} 1143 \mathrm{GJB}^{+/+/}$xenografts $(\mathrm{n}=5)$ and $\mathrm{HCC} 1143 \mathrm{GJB}^{+/++}$xenografts $(\mathrm{n}=4)$. The black line indicates mean adipocyte area. Each point represents individual adipocyte. (F) Kaplan-Meier analysis of tumor onset of mice bearing indicated $\mathrm{Cx} 31$ partial knockout orthotopic xenografts ( $\mathrm{n}=5$ per group) and treated with vehicle or with $1 \mathrm{mg} / \mathrm{kg}$ CL316273. For (C) and (D) mean \pm s.e.m. is shown. $* \mathrm{P}<0.05,{ }^{*} \mathrm{P}<0.01,{ }^{* * *} \mathrm{P}<0.001,{ }^{*} * * \mathrm{P}<0.0001$; unpaired two-tailed $\mathrm{t}$ test (A), (C), and (D), log-rank test (B) and (F). 
A

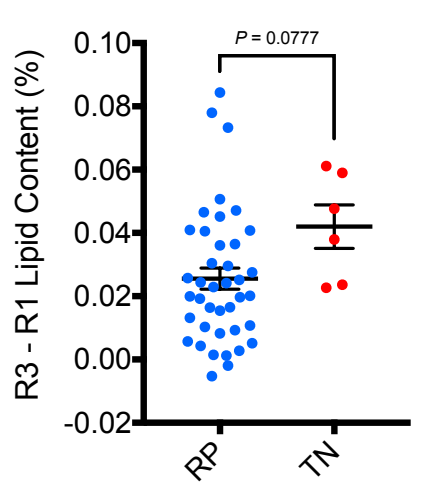

B

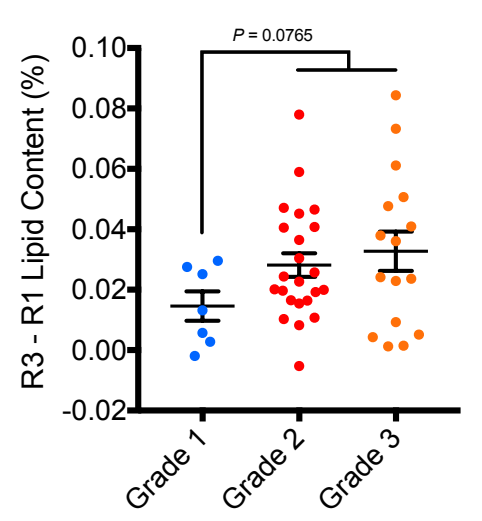

C

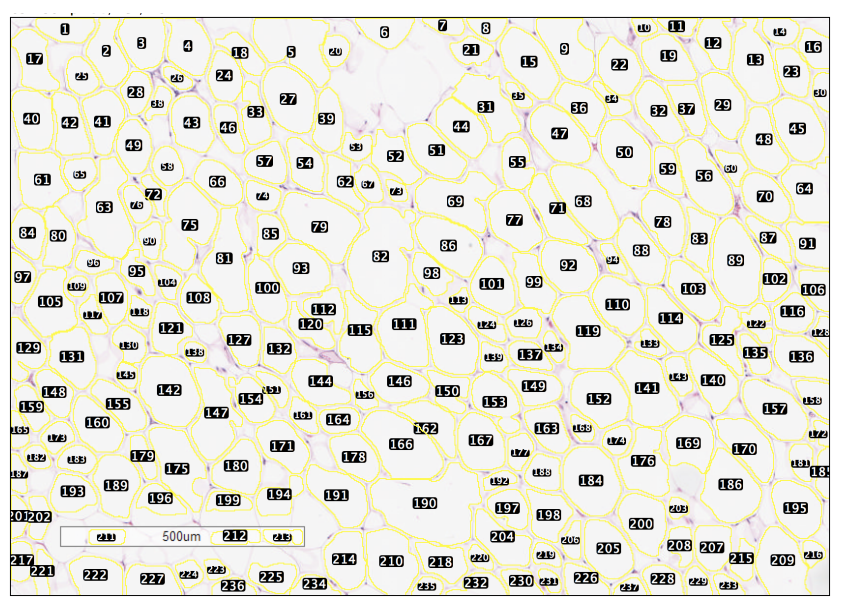


Camarda R, et al. 2018 - preprint version -www.biorxiv.org

\section{Supplemental Figure Legend:}

Fig. S1. NAT lipid content by receptor status and tumor grade, and adipocyte area quantification. (A) Change in lipid content in R3 of NAT versus R1 of NAT from TN and RP patients. (B) Change in lipid content in R3 of NAT versus R1 of NAT from grade 1, 2 and 3 patients. (C) Example of Adiposoft software output on manual mode before curation to identify whole, individual adipocytes. $\mathrm{P}$ values indicated; unpaired two-tailed t test (A) and (B).

\section{Supplementary Table Legends:}

Table S1. Patient ID, receptor status, histological section availability, percent lipid content (lipid content $/$ lipid + water + protein content) of L, R1, R2 and R3, and Scarff-Bloom-Richardson (SBR) grade from patients $(\mathrm{n}=46)$ with invasive breast cancer.

Table S2. LC-MS/MS of LCM samples from 75 patients with invasive breast cancer. (A) Sample number, ID number, tissue type, and tumor subtype (when applicable) of 75 patients. (B) Spectral counts of proteins detected via LC-MS/MS from samples in (A).

Table S3. RNA expression changes in MTB-TOM tumors $(\mathrm{n}=11)$ compared to non-tumor mammary glands $(\mathrm{n}=3)$. Differential expression analysis was performed using the 'limma' R package (17). 